\title{
Value of F-18-fluorodeoxyglucose positron emission tomography after induction therapy of locally advanced bronchogenic carcinoma
}

\author{
Dirk Hellwig, MD, Dipl-Phys ${ }^{a}$ \\ Thomas P. Graeter, MD ${ }^{\mathrm{b}}$ \\ Dieter Ukena, MD, $\mathrm{PhD}^{\mathrm{c}}$ \\ Thomas Georg, PhD ${ }^{\mathrm{d}}$ \\ Carl-Martin Kirsch, MD, PhD, Dipl-Ing ${ }^{\text {a }}$ \\ Hans-Joachim Schäfers, MD, PhD ${ }^{\mathrm{b}}$
}

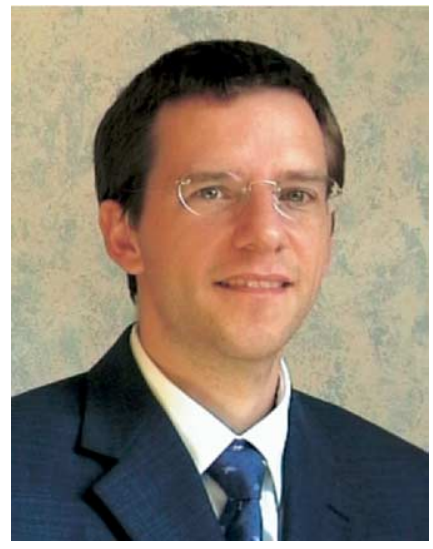

Dr Hellwig
From the Departments of Nuclear Medicine, ${ }^{\mathrm{a}}$ Thoracic and Cardiovascular Surgery, ${ }^{\mathrm{b}}$ Internal Medicine V (Pneumology), ${ }^{\mathrm{c}}$ and the Institute of Medical Biometrics, Epidemiology, and Medical Informatics, ${ }^{d}$ University Hospital Homburg, Homburg/ Saar, Germany.

Read at the Eighty-fourth Annual Meeting of The American Association for Thoracic Surgery, Toronto, Ontario, Canada, April 25-28, 2004.

Received for publication April 20, 2004; revisions received July 6, 2004; accepted for publication July 13, 2004.

Address for reprints: Thomas P. Graeter, MD, Department of Thoracic and Cardiovascular Surgery, University Hospital Homburg, D-66421 Homburg/Saar, Germany (E-mail: Thomas.Graeter@uniklinik-saarland.de).

J Thorac Cardiovasc Surg 2004;128:892-9

0022-5223/\$30.00

Copyright (C) 2004 by The American Association for Thoracic Surgery

doi:10.1016/j.jtcvs.2004.07.031
Objectives: Induction therapy is an important treatment option in locally advanced non-small cell lung cancer. F-18-fluorodeoxyglucose positron emission tomography (FDG-PET) has an important role in initial staging. The aim of this study was to assess the value of FDG-PET in restaging after induction therapy and in analyzing tumor viability, nodal status, distant metastases, and prognosis.

Methods: Forty-seven patients with locally advanced non-small cell lung cancer accepted for resection after induction therapy underwent FDG-PET. Images were interpreted visually for mediastinal nodal status and metastatic spread. The FDG accumulation in the tumor site was measured by using the maximum standardized uptake value.

Results: Unexpected metastases were detected by means of FDG-PET in 9 patients. Surgical intervention was not performed in 8 patients with confirmed metastases. The rate of unexpected findings increased from complete radiologic remission $(0 \%)$ over partial remission $(9 \%)$ to no change $(67 \%)$. The standardized uptake value was higher in tumors with $(\mathrm{n}=26)$ than in those without $(\mathrm{n}=11)$ histologic proof of viability $(6.4 \pm 5.3$ vs $2.9 \pm 1.6, P=.006)$. All patients with standardized uptake values of greater than 5.8 had viable tumors. Sensitivity, specificity, and negative predictive value were $81 \%, 64 \%$, and $58 \%$ for tumor viability and $50 \%, 88 \%$, and $85 \%$ for persistent mediastinal disease. Median survival after resection was greater than 56 months for patients with tumor standardized uptake values of less than 4 and 19 months for patients with standardized uptake values of 4 or greater $(P<.001)$.

Conclusion: FDG-PET helps in the selection of patients for resection after induction therapy. It can be used to detect unexpected distant metastases, especially after poor response to induction therapy. Its high negative predictive value in mediastinal restaging allows for omission of repeat mediastinoscopy. Tumor standardized uptake value after induction is a prognostic factor.

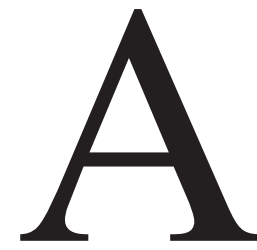

lthough approximately one third of patients with lung cancer can be treated with primary resection, the majority present in an advanced stage. Induction therapy with combinations of chemotherapy and radiotherapy has been proved to be efficient in locally advanced stages of non-small cell lung cancer (NSCLC). It appears to improve prognosis, and different protocols are currently being investigated in prospective trials. ${ }^{1}$

The response to neoadjuvant therapy is commonly assessed by means of anatomic imaging to document the change in size of malignant lesions. This fails to 
provide information on the biologic behavior of residual masses (eg, tumor viability). In laboratory studies the response to chemotherapy or radiotherapy was detected earlier on the basis of changes in glucose metabolism rather than on the basis of size regression. ${ }^{2}$

Positron emission tomography (PET) with F-18fluorodeoxyglucose (FDG) visualizes the glucose metabolism in vivo and is increasingly used in oncologic staging. ${ }^{3}$ The role of FDG-PET has been established in the initial staging of lung cancer. ${ }^{4-6}$

Until now, only limited data were published on the performance of FDG-PET in patients with lung cancer after induction therapy. Previous studies reported the potential ability of FDG-PET to detect residual tumor viability, ${ }^{7-11}$ persistent mediastinal disease, ${ }^{7-9,11,12}$ and distant metastases. ${ }^{8}$ For the selection of patients about to undergo resection after induction, it is essential to exclude distant metastases. To our knowledge, there are no published data on the rate of unexpected distant metastases detected by means of FDG-PET for restaging. Even if FDG-PET provides prognostic information after initiation of palliative chemotherapy ${ }^{13}$ or completion of conformal (chemo)radiotherapy, ${ }^{14,15}$ there are no reports on the prognostic relevance of the preoperative FDG uptake in the residual tumor after induction therapy of NSCLC.

Thus, we investigated the value of FDG-PET after completion of induction therapy. We did a comparison with respect to unexpected distant metastases, viability of the residual tumor, accuracy of nodal restaging, and postoperative survival.

\section{Patients and Methods \\ Patients}

The patient population consisted of 47 patients (32 men) with histologically proved NSCLC. They were accrued from July 1998 through September 2003 at the University Hospital Homburg. All patients were recruited from ongoing prospective studies investigating the use of multimodal treatment of locally advanced NSCLC and were accepted for resection before FDG-PET.

The patients' median age was 60 years (range, 37-77 years). Initial stages were IIB (T3 N0, n = 3), IIIA (T1-3 N2, n = 10) and IIIB $(\mathrm{n}=34)$ defined by T2-3 N3 $(\mathrm{n}=9)$, or T4 $(\mathrm{n}=25)$. Histology of the tumor was squamous cell carcinoma $(n=24)$, adenocarcinoma $(n=20)$, large cell carcinoma $(n=1)$, mucoepidermoid carcinoma $(n=1)$, and unspecified NSCLC $(n=1)$.

All patients underwent initial staging procedures according to current German recommendations, ${ }^{16}$ including anterior cervical mediastinoscopy in 16 patients with suspected mediastinal lymph node metastases. In 34 cases FDG-PET imaging had been performed before therapy in the initial staging.

Tumor-specific therapy consisted of platinum-based cytostatic chemotherapy in all but 1 patient with additional irradiation of the tumor site and involved the mediastinum in 33 patients (45-68 Gy, 1-1.5 Gy/d, in 19 patients; $32 \mathrm{~Gy}, 2 \times 2 \mathrm{~Gy} / \mathrm{d}$, in 14 patients). One patient with initial stage $\mathrm{T} 3 \mathrm{pN} 0 \mathrm{M} 0$ disease received preoperative irradiation without chemotherapy (48.6 Gy).

\section{Assessment of Tumor Response}

Treatment response of the primary tumor was documented by means of computed tomographic (CT) imaging and, with invaded airways, bronchoscopy after completion of the induction therapy by using World Health Organization criteria for definition of complete response, partial response, no change, and progressive disease. By means of conventional staging, 3 patients had a complete response, 35 had a partial response, and 9 had no change.

\section{PET Imaging}

All patients had whole-body FDG-PET imaging within a median of 5 weeks (range, 2-11 weeks) after completion of induction therapy. After overnight fasting, the blood glucose level was determined to ascertain a level of less than $160 \mathrm{mg} / \mathrm{dL}$. The patients then received $250 \mathrm{MBq}$ (range, 160-350 MBq) of 2-deoxy-2-[F-18]fluoro-Dglucose (FDG purchased from Forschungszentrum Karlsruhe, Germany, or f-con, Rendsburg, Germany). Imaging started 90 minutes after injection and was performed as previously reported. ${ }^{17}$ The images were visually interpreted for uptake in the primary tumor and mediastinal lymph nodes and for evidence of metastatic spread. Two board-certified nuclear medicine physicians analyzed film documentations in all 3 planes and maximum intensity projections in cine mode. Semiquantitative evaluation was based on standardized uptake values (SUVs) calculated as the ratio of measured activity concentration over an assumed homogenous distribution of the applied radioactivity in the whole body. The peak SUV in the tumor site was measured by using a region-of-interest technique with the standard image analysis software of the PET scanner (ECAT version 7.2). For evaluation of the mediastinal lymph nodes, circular regions of interest with a diameter of $1 \mathrm{~cm}$ were defined in the hilar and mediastinal lymph node stations according to the method of Mountain and Dresler, ${ }^{18}$ and the peak SUV was measured. Lesions with SUVs of greater than 2.5 were considered malignant because this value usually exceeds the normal level in the mediastinal blood pool. ${ }^{19}$

\section{Surgical Intervention}

In 37 patients resection and systematic lymph node dissection was performed after induction therapy within a median of 2 weeks (range, 1-6 weeks) after the FDG-PET scan. A typical lobectomy was performed in 17 patients, and 3 patients underwent lobectomy and chest wall resection. A sleeve lobectomy was required in 12 patients, and 3 had a bronchovascular sleeve resection, reflecting our policy of avoidance of pneumonectomy in central tumors. ${ }^{20}$ Pneumonectomy was necessary in 2 patients. All of these procedures were combined with a systematic lymphadenectomy. Routine pathologic examination of the surgical specimens was performed according to current recommendations. ${ }^{21}$

\section{Statistics}

Numerical values are reported as medians with ranges and are compared between groups by the Wilcoxon $U$ test. Proportions are reported as percentages with $95 \%$ confidence intervals (95\% CIs). The frequency of unexpected distant metastases for patients with no change, a partial response, and no change was compared by using the Somers D test. The difference in patients with and without a previous PET scan was analyzed with the Fisher exact test. Contingency tables of true-negative, false-negative, true-positive, and false-positive findings have been constructed for the 


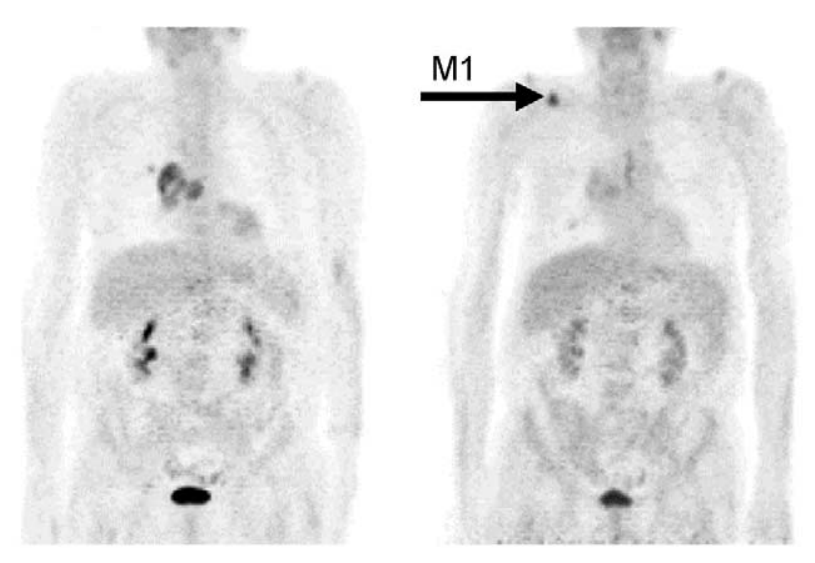

Figure 1. Initial (left) and restaging (right) FDG-PET of a patient with an unexpected soft tissue metastasis after inductive chemoradiotherapy of an adenocarcinoma of the right lower lobe (initial stage T4 pN2 M0). Conventional restaging showed partial remission, but the planned tumor resection was cancelled after histologic confirmation of malignancy in the FDG-accumulating lesion in the right shoulder.

detection of expected metastases, the viability of residual tumor, and lymph node staging. Diagnostic performance parameters (sensitivity, specificity, accuracy, negative predictive value [NPV], and positive predictive value) were derived from these contingency tables. The McNemar test was used to compare diagnostic performance parameters between different diagnostic modalities.

Survival data were obtained from follow-up contacts or from telephone interviews of the patients' local physicians. The observation period ended in December 2003. Survival was analyzed for patients without distant metastases who underwent resection. Survival time was calculated with the Kaplan-Meier method. Survival differences between groups were compared by using the log-rank test.

\section{Results}

\section{Unexpected Metastases}

After induction therapy, FDG-PET showed findings consistent with unexpected metastases in 9 instances ( 2 liver, 1 bone, 1 adrenal, 1 spleen, 1 soft tissue, 1 thoracic wall lymph node, 1 supraclavicular lymph node, and 1 axillary lymph node). In 8 patients the findings were confirmed by means of histology $(n=4)$ or serial imaging $(n=4)$. These patients did not undergo resection. A typical case is illustrated in Figure 1. The rate of unexpected confirmed metastases was 8 of 47 (prevalence 17\%). In 1 patient the false-positive FDG accumulation in an axillary lymph node could not be confirmed as being caused by malignancy. One false-negative finding occurred in a patient with a brain metastasis outside the field of view of the PET scan, which became symptomatic after the restaging procedures. No significant difference was found for the frequency of distant metastases in patients with (4/34 [15\%]) or without (4/13 [31\%]) an FDG-PET scan for initial staging $(P=.198)$.
There was a significant correlation between the frequency of unexpected metastases detected by means of FDG-PET and the tumor response evaluated by conventional imaging (Figure 2). The rate increases from complete response over partial response to no change $(P=.003)$.

\section{Viability of Residual Tumors}

Resection of the primary tumor was performed in 37 patients. Two patients refused surgical intervention.

In the 2 patients with complete response (determined by conventional imaging criteria), no viable tumor cells were found in the specimen. Two of 3 patients with no change had viable tumor cells. In patients with a partial response, 24 had residual tumor cells, and in 8 patients no viable tumor was found. Overall, tumor viability was seen in the specimens in 26 of 37 surgical patients. In 11 patients there was no histologic proof of a viable tumor.

The glucose metabolism in viable residual tumors was higher $(P=.006)$ than in tumors without histologic proof of viable cells (Table 1). All primary tumors with an SUV of 5.8 or more contained viable tumor cells. PET findings were false negative in 5 instances of microscopic tumor residual tumor $(\mathrm{n}=4)$ and bronchioloalveolar carcinoma $(\mathrm{n}=1)$. False-positive PET findings occurred after irradiation $(\mathrm{n}=$ 3 ) or for a brief period (17 days) after the last chemotherapy cycle $(\mathrm{n}=1)$. Diagnostic test parameters for viability with an SUV threshold of 2.5 were a sensitivity of $81 \%(95 \% \mathrm{CI}$, $61 \%-93 \%$ ), a specificity of $64 \%$ (95\% CI, 31\%-89\%), an accuracy of $76 \%$ (95\% CI, 59\%-88\%), a positive predictive value of $84 \%(95 \% \mathrm{CI}, 64 \%-95 \%)$, and an NPV of $58 \%$ (95\% CI, 28\%-85\%).

\section{Assessment of Mediastinal Nodal Status}

A total of 131 lymph node stations were removed during thoracotomy (3.6 per patient), including 78 mediastinal stations. Viable tumor cells were found in 11 samples (median SUV, 3.8; range, 2.0-8.9) of 8 patients. Visual interpretation of FDG-PET resulted in 7 true-positive, 4 false-negative, 64 truenegative, and 3 false-positive findings for the mediastinal lymph node stations. Using quantitative criteria, assuming malignancy in lymph nodes with SUVs of greater than 2.5, we observed 7 true-positive, 4 false-negative, 53 true-negative, and 14 false-positive findings. This approach did not increase the number of detected lymph node metastases compared with quantitative analysis but rather increased the rate of falsepositive findings.

The patient-based analysis showed that visual interpretation of FDG-PET correctly detected residual mediastinal disease in 4 patients or excluded residual mediastinal disease in 22 patients. In 4 patients the presence of solitary mediastinal lymph node involvement was missed, and falsepositive findings occurred in 3 patients. Two patients were excluded from analysis because of incomplete mediastinal 


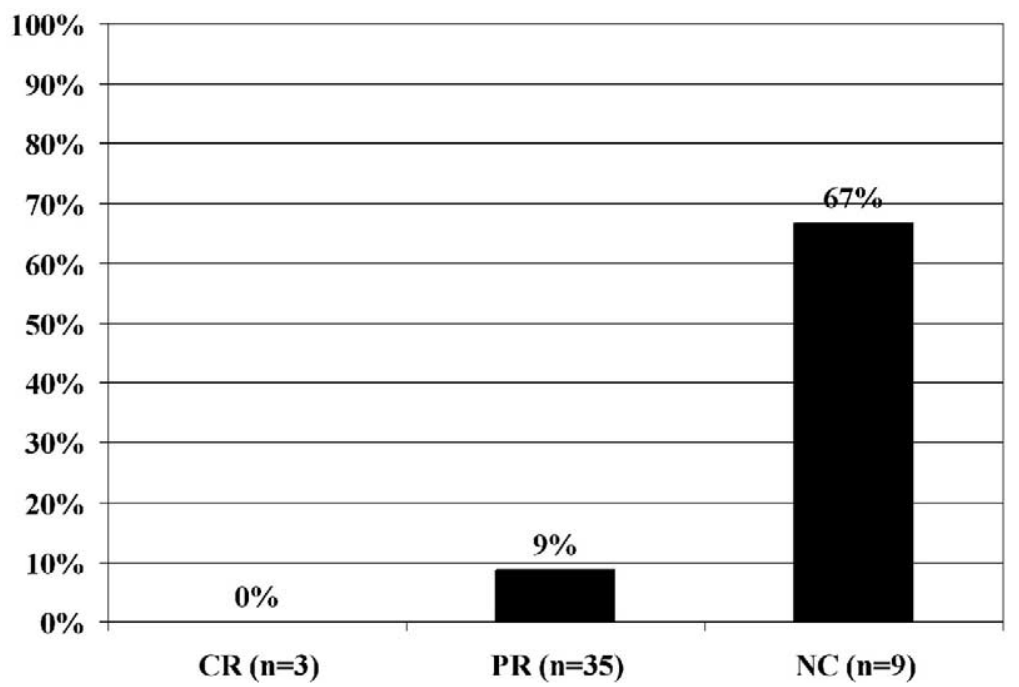

Figure 2. Rate of unexpected distant metastases for patients with complete response (CR), partial response (PR), and no change (NC). The frequency of unexpected findings correlates inversely with the tumor response, as evaluated by means of conventional imaging.

lymph node biopsies not covering PET-positive contralateral nodes. The diagnostic test parameters for the patientbased analysis and for qualitative and quantitative nodal analysis are summarized in Table 2. Although we observed no difference in the sensitivities of quantitative nodal analysis, the specificity of visual interpretation is higher than that of quantitative analysis of lymph node involvement by means of FDG-PET (McNemar test, $P=.001$ ).

\section{Survival}

The median follow-up duration was 36 months (range, 1-63 months). Two patients were lost to follow-up since discharge from the hospital after surgical intervention. During the observation period, 14 of the 35 remaining patients died. The estimated median survival for the total group was 38 months from the date of the operation. The estimated median survival (Figure 3) was longer for patients with SUVs of less than 4 in the residual tumor than for those with SUVs of 4 or greater ( $>56$ months vs 19 months, $P<.001$ ).

\section{Discussion}

Multimodal treatment concepts have become a therapeutic option for patients with stage IIIA NSCLC. Even in patients with stage IIIB disease, current investigations indicate that operations after induction treatment can prolong survival. ${ }^{22}$ Different combinations of induction therapy with chemotherapy, radiotherapy, or both, followed by surgical resection, are currently under investigation. ${ }^{1}$

The reevaluation of tumor viability and metastatic spread remains a challenge for anatomic imaging because the reduction of tumor size, which does not occur immediately after cell death, is used as a diagnostic criterion. The use of
TABLE 1. FDG accumulation reported as median SUV in primary tumor sites after completion of induction therapy

\begin{tabular}{lcllll}
\hline \multirow{2}{*}{$\begin{array}{l}\text { Response from } \\
\text { CT imaging }\end{array}$} & \multicolumn{2}{c}{ Viable tumor } & & \multicolumn{2}{c}{ No viable tumor cells } \\
\cline { 2 - 3 } \cline { 5 - 6 } & SUV & Patients & & SUV & Patients \\
\hline CR & NA & $n=0$ & & $1.9(1.7-2.2)$ & $\mathrm{n}=2$ \\
PR & $4.7(2.0-21.6)$ & $\mathrm{n}=24$ & & $2.0(1.2-5.8)$ & $\mathrm{n}=8$ \\
NC & $11.8(8.3-15.2)$ & $\mathrm{n}=2$ & & 3.5 & $\mathrm{n}=1$ \\
All patients & $4.8(2.0-21.6)$ & $\mathrm{n}=26$ & & $2.2(1.2-5.8)$ & $\mathrm{n}=11$
\end{tabular}

Figures in parentheses are ranges. FDG, F-18-fluorodeoxyglucose; SUV, standardized uptake value; $C T$, computed tomographic; $C R$, complete response; $N A$, not applicable; $P R$, partial response; $N C$, no change.

TABLE 2. Diagnostic test parameters for mediastinal lymph node restaging for different types of analysis

\begin{tabular}{lccc}
\hline Test & $\begin{array}{c}\text { Patient based, } \\
\text { visual }\end{array}$ & $\begin{array}{c}\text { Nodal analysis, } \\
\text { quantitative }\end{array}$ & $\begin{array}{c}\text { Nodal analysis, } \\
\text { visual }\end{array}$ \\
\hline Sensitivity (\%) & $50(16-84)$ & $64(31-89)$ & $64(31-89)$ \\
Specificity (\%) & $88(69-97)$ & $79(67-88)$ & $96(87-99)$ \\
Accuracy (\%) & $79(61-91)$ & $77(66-86)$ & $91(82-96)$ \\
PPV (\%) & $57(18-90)$ & $33(15-57)$ & $70(35-93)$ \\
NPV (\%) & $85(65-96)$ & $93(83-98)$ & $94(86-98)$ \\
\hline
\end{tabular}

Patient-based analysis evaluates whether FDG-PET correctly assigns the $\mathrm{N}$ stage with residual mediastinal involvement and compares the stages N0/N1 versus N2/N3 for each patient. Nodal analysis is used for comparison of F-18-fluorodeoxyglucose positron emission tomography findings with histopathology in each mediastinal biopsy specimen. Proportions are reported as percentage values with lower and upper bounds of the $95 \%$ confidence interval. PPV, Positive predictive value; NPV, negative predictive value. 


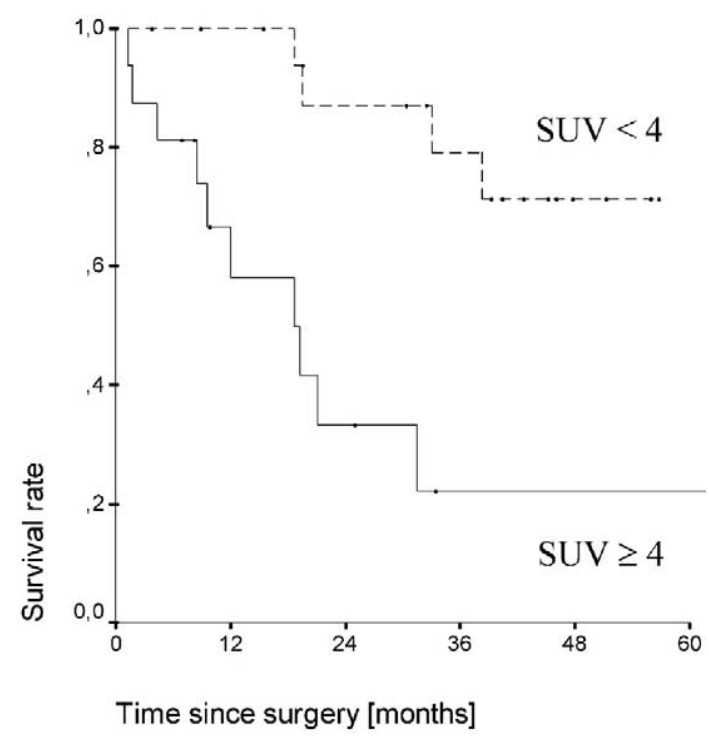

Figure 3. Survival probability data for patients after resection of residual tumor after induction therapy. The survival of patients with an FDG uptake in the residual primary tumor with an SUV of 4 or greater is shorter than for patients with SUVs of less than 4 (log-rank test, $P=.0006)$.

surgical mediastinal restaging by means of repeat mediastinoscopy is limited because it remains incomplete in up to $40 \%$ of patients because of fibrosis and adhesions. ${ }^{22}$

FDG-PET has been shown to be the most accurate noninvasive imaging modality in the initial staging of NSCLC. ${ }^{4}$ The role of PET in the restaging of NSCLC is not yet clear. Several clinical studies focusing on single aspects of PET have been published. ${ }^{7-14}$ Conflicting data have been published regarding sensitivity and specificity in mediastinal lymph node involvement, ${ }^{7-9,11,12}$ and the prognostic effect of PET results is unclear.

One important ability of FDG-PET is the detection of unexpected distant metastases. Consistent with the study of Akhurst and colleagues, ${ }^{8}$ we were able to detect all distant metastases after induction therapy. Unexpected metastases were present in $17 \%$ of our patients, some of them at unusual sites (eg, soft tissue, spleen, and thoracic wall lymph node). These are not covered by recommended imaging procedures ${ }^{16}$ or easily missed on routine $\mathrm{CT}$ scan interpretation.

It might be argued that these metastases had been present already before induction treatment and should have been detected during pretreatment PET scanning. We did not perform a PET investigation in all patients before induction, but there was no difference in the frequency of unexpected metastases between patients with or without an initial PET scan. Therefore the lesions might be of more recent origin. The incidence of $17 \%$ in our cohort is similar to the FDGPET results of unexpected distant metastases in $20 \%$ of patients with NSCLC to undergo radical radiotherapy. ${ }^{23}$ The rate has been shown to increase with pre-PET clinical stage from $8 \%$ for stage I through $18 \%$ for stage II to $24 \%$ for stage III. ${ }^{23}$ Similarly, we observed an increasing frequency of distant metastases with poor tumor response, as assessed on the basis of conventional size criteria. This reached an incidence of $67 \%$ in patients with a clinical response of no change.

Regarding the assessment of tumor viability, our results are in line with previous reports on FDG uptake after induction therapy of NSCLC. ${ }^{7-9,11}$ Akhurst and colleagues ${ }^{8}$ reported a sensitivity of $90 \%$ and a specificity of $67 \%$ for the detection of tumor viability by means of FDG-PET. Similarly, Cerfolio and coworkers ${ }^{9}$ found corresponding values of $97 \%$ and $100 \%$, and Ryu and associates ${ }^{7}$ documented values of $88 \%$ and $67 \%$. In the most recent report a sensitivity of only 58\% and a specificity of $100 \%$ are published for FDG-PET performed 2 weeks after the completion of chemotherapy. ${ }^{11}$

In our series we mainly observed false-positive findings after radiotherapy. Irradiation is well known to induce such changes. ${ }^{24}$ FDG-PET reading was compared with a recent CT image to minimize the chance of misinterpretation. On the other hand, we saw false-negative findings, primarily in small tumors and bronchioloalveolar carcinoma. Similar observations have been published for the initial evaluation of lung lesions. ${ }^{4}$ The sensitivity of FDG-PET in restaging is limited by the size of malignant tissue required for detection, whereas histologically, the existence of only a single tumor cell can be regarded as a sign of viability.

For mediastinal restaging, we analyzed the lymph node stations by means of visual interpretation, as well as quantitative measurement. The specificity of visual interpretation was higher than that of quantitative analysis, without any difference in sensitivity. This can be explained by the fact that small deviations in mediastinal blood pool radioactivity can exceed the SUV threshold of 2.5, which can lead to false-positive findings. The visual analysis additionally uses the shape of activity distribution, interpreting lesions of focally increased FDG uptake as malignant.

In a previous investigation on FDG-PET and mediastinoscopy during initial staging of lung cancer, we found a median SUV of 7.3 (range, 1.6-18.9) in metastatic mediastinal lymph nodes. ${ }^{17}$ This was higher than the SUV in lymph node metastases after induction therapy in this study (median SUV, 3.8; range, 2.0-8.9; $P=.046$ ). The difference might be due to decreased metabolism or the amount of malignant tissue. These factors might also explain the reduced sensitivity of PET.

Our results in mediastinal restaging by means of FDGPET are similar in diagnostic performance to the data published by Akhurst and colleagues ${ }^{8}$ and Ryu and associates. ${ }^{7}$ We cannot confirm the observation of Cerfolio and cowork- 
ers $^{9}$ that PET results are ideal for the subgroup of paratracheal lymph nodes because we observed 2 false-negative findings in the paratracheal and 2 false-negative findings in the infracarinal stations. Nevertheless, the overall diagnostic performance of their study compares with that of ours.

The results of PET have to be compared with those published for repeat mediastinoscopy. After initial mediastinoscopy and induction treatment, this procedure can be technically difficult. Because of inflammatory changes, lymph nodes might be difficult to locate. ${ }^{25,26}$ Repeat mediastinoscopy has been shown to result in a sensitivity of only $50 \%$ after induction therapy of stage IIIB NSCLC. ${ }^{22}$ The NPV was reported to be $71 \%$. This is similar to the $85 \%$ NPV of FDG-PET with respect to mediastinal lymph node metastases in our patient-based analysis.

FDG uptake in NSCLC has been identified as a prognostic parameter at initial presentation, ${ }^{6,27,28}$ early after the initiation of chemotherapy, ${ }^{13}$ during radiotherapy, or after completion of tumor treatment. ${ }^{14,29,30}$ Our analysis is the first to show that the preoperative FDG uptake in the residual tumor after induction is a prognostic parameter with a significant correlation to survival.

There are several potential limitations of our study in that it is a retrospective investigation, and the number of patients studied is limited. Because surgical treatment of stage III NSCLC is not standard treatment, the accrued patients were selected from ongoing studies. This explains a high proportion of individuals with initial stage IIIB disease and the use of different induction schemes.

In left-sided resections lymph node dissection only reached ipsilateral hilar and mediastinal nodes, so that there is no pathologic analysis of the contralateral lymph node stations.

The timing of the FDG-PET and the completion of induction varied between patients, with a median interval of 5 weeks and a range of 2 to 11 weeks. Presently, there are no systematic data available on patients with NSCLC to determine the optimal time to assess residual malignant tissue after induction therapy. The interval, however, might have an effect on sensitivity. Port and coworkers ${ }^{11}$ used an interval of 2 weeks after induction chemotherapy and found the lowest sensitivity of all studies in this field.

As a recommendation for the time point of repeat FDGPET, we suggest an interval of at least 2 weeks after completion of induction therapy to achieve a sufficient sensitivity in the detection of residual viable tumor and to avoid inflammatory therapy-induced reactions.

FDG-PET could become the standard of care after induction therapy because it helps in the selection of patients for resection caused by the detection of unexpected distant metastases, especially in patients with poor response. Compared with initial staging, the sensitivity in the detection of the primary tumor and of lymph node metastases is slightly reduced. The high NPV of FDG-PET in mediastinal restaging justifies omis- sion of repeat mediastinoscopy if the mediastinum is PET negative. After induction therapy, the preoperative FDG uptake in NSCLC provides a prognostic parameter.

\section{References}

1. Thomas M, Baumann M, Deppermann M, Freitag L, Gatzemeier U, Huber R, et al. [Recommendations on the therapy of bronchial carcinoma]. Pneumologie. 2002;56:113-31.

2. Iosilevsky G, Front D, Bettman L, Hardoff R, Ben-Arieh Y. Uptake of gallium-67 citrate and [2-3H]deoxyglucose in the tumor model, following chemotherapy and radiotherapy. J Nucl Med. 1985;26:278-82.

3. Reske SN, Kotzerke J. FDG-PET for clinical use. Results of the 3rd German Interdisciplinary Consensus Conference, "Onko-PET III," 21 July and 19 September 2000. Eur J Nucl Med. 2001;28:1707-23.

4. Hellwig D, Ukena D, Paulsen F, Bamberg M, Kirsch CM. [Metaanalysis of the efficacy of positron emission tomography with F-18fluorodeoxyglucose in lung tumors. Basis for discussion of the German Consensus Conference on PET in Oncology 2000]. Pneumologie. 2001;55:367-77.

5. van Tinteren H, Hoekstra OS, Smit EF, van den Bergh JH, Schreurs AJ, Stallaert RA, et al. Effectiveness of positron emission tomography in the preoperative assessment of patients with suspected non-smallcell lung cancer: the PLUS multicentre randomised trial. Lancet. 2002;359:1388-93.

6. Vansteenkiste JF, Stroobants SG, Dupont PJ, De Leyn PR, Verbeken EK, Deneffe GJ, et al. Prognostic importance of the standardized uptake value on (18)F-fluoro-2-deoxy-glucose-positron emission tomography scan in non-small-cell lung cancer: an analysis of 125 cases. Leuven Lung Cancer Group. J Clin Oncol. 1999;17:3201-6.

7. Ryu JS, Choi NC, Fischman AJ, Lynch TJ, Mathisen DJ. FDG-PET in staging and restaging non-small cell lung cancer after neoadjuvant chemoradiotherapy: correlation with histopathology. Lung Cancer. 2002;35:179-87.

8. Akhurst T, Downey RJ, Ginsberg MS, Gonen M, Bains M, Korst R, et al. An initial experience with FDG-PET in the imaging of residual disease after induction therapy for lung cancer. Ann Thorac Surg. 2002;73:259-66.

9. Cerfolio RJ, Ojha B, Mukherjee S, Pask AH, Bass CS, Katholi CR. Positron emission tomography scanning with 2-fluoro-2-deoxy-d-glucose as a predictor of response of neoadjuvant treatment for non-small cell carcinoma. J Thorac Cardiovasc Surg. 2003;125:938-44.

10. Choi NC, Fischman AJ, Niemierko A, Ryu JS, Lynch T, Wain J, et al. Dose-response relationship between probability of pathologic tumor control and glucose metabolic rate measured with FDG PET after preoperative chemoradiotherapy in locally advanced non-small-cell lung cancer. Int J Radiat Oncol Biol Phys. 2002;54:1024-35.

11. Port JL, Kent MS, Korst RJ, Keresztes R, Levin MA, Altorki NK. Positron emission tomography scanning poorly predicts response to preoperative chemotherapy in non-small cell lung cancer. Ann Thorac Surg. 2004;77:254-9.

12. Vansteenkiste JF, Stroobants SG, De Leyn PR, Dupont PJ, Verbeken EK. Potential use of FDG-PET scan after induction chemotherapy in surgically staged IIIa-N2 non-small-cell lung cancer: a prospective pilot study. The Leuven Lung Cancer Group. Ann Oncol. 1998;9: 1193-8.

13. Weber WA, Petersen V, Schmidt B, Tyndale-Hines L, Link T, Peschel $\mathrm{C}$, et al. Positron emission tomography in non-small-cell lung cancer: prediction of response to chemotherapy by quantitative assessment of glucose use. J Clin Oncol. 2003;21:2651-7.

14. MacManus MP, Hicks RJ, Matthews JP, McKenzie A, Rischin D, Salminen EK, et al. Positron emission tomography is superior to computed tomography scanning for response-assessment after radical radiotherapy or chemoradiotherapy in patients with non-small-cell lung cancer. J Clin Oncol. 2003;21:1285-92.

15. Ichiya Y, Kuwabara Y, Sasaki M, Yoshida T, Omagari J, Akashi Y, et al. A clinical evaluation of FDG-PET to assess the response in radiation therapy for bronchogenic carcinoma. Ann Nucl Med. 1996;10:193200 . 
16. Thomas M, Gatzemeier U, Goerg R, Matthiessen W, Morr H, Schonfeld N, et al. [Recommendations on the diagnosis of bronchial carcinoma. German Pneumology Society]. Pneumologie. 2000;54:361-71.

17. Graeter TP, Hellwig D, Hoffmann K, Ukena D, Kirsch CM, Schäfers HJ. Mediastinal lymph node staging in suspected lung cancer: comparison of positron emission tomography with F-18-fluorodeoxyglucose and mediastinoscopy. Ann Thorac Surg. 2003;75:231-6.

18. Mountain CF, Dresler CM. Regional lymph node classification for lung cancer staging. Chest. 1997;111:1718-23.

19. Ramos CD, Erdi YE, Gonen M, Riedel E, Yeung HW, Macapinlac HA, et al. FDG-PET standardized uptake values in normal anatomical structures using iterative reconstruction segmented attenuation correction and filtered back-projection. Eur J Nucl Med. 2001;28:155-64.

20. Lausberg HF, Graeter TP, Wendler O, Demertzis S, Ukena D, Schäfers HJ. Bronchial and bronchovascular sleeve resection for treatment of central lung tumors. Ann Thorac Surg. 2000;70:367-72.

21. Gibbs AR, Attanoos RL. Examination of lung specimens. J Clin Pathol. 2000;53:507-12.

22. Pitz CC, Maas KW, Van Swieten HA, de la Riviere AB, Hofman P, Schramel FM. Surgery as part of combined modality treatment in stage IIIB non-small cell lung cancer. Ann Thorac Surg. 2002;74:164-9.

23. MacManus MP, Hicks RJ, Matthews JP, Hogg A, McKenzie AF, Wirth A, et al. High rate of detection of unsuspected distant metastases by pet in apparent stage III non-small-cell lung cancer: implications for radical radiation therapy. Int J Radiat Oncol Biol Phys. 2001;50:287-93.

24. Nestle U, Hellwig D, Fleckenstein J, Walter K, Ukena D, Rübe C, et al. Comparison of early pulmonary changes in 18FDG-PET and CT after combined radiochemotherapy for advanced non-small-cell lung cancer: a study in 15 patients. In: Dörr W, Engenhart-Cabillic R, Zimmermann J. Normal tissue reactions in radiotherapy and oncology. Frontiers of Radiation Therapy and Oncology. Vol. 37. Basel: Karger; 2002. p. 26-33.

25. Roberts JR, Eustis C, Devore R, Carbone D, Choy H, Johnson D. Induction chemotherapy increases perioperative complications in patients undergoing resection for non-small cell lung cancer. Ann Thorac Surg. 2001;72:885-8.

26. Doddoli C, Thomas P, Thirion X, Serée Y, Giudicelli R, Fuentes P. Postoperative complications in relation with induction therapy for lung cancer. Eur J Cardiothorac Surg. 2001;20:385-90.

27. Ahuja V, Coleman RE, Herndon J, Patz EF Jr. The prognostic significance of fluorodeoxyglucose positron emission tomography imaging for patients with nonsmall cell lung carcinoma. Cancer. 1998;83:918-24.

28. Jeong HJ, Min JJ, Park JM, Chung JK, Kim BT, Jeong JM, et al. Determination of the prognostic value of [(18)F]fluorodeoxyglucose uptake by using positron emission tomography in patients with nonsmall cell lung cancer. Nucl Med Commun. 2002;23:865-70.

29. Higashi K, Ueda Y, Arisaka Y, Sakuma T, Nambu Y, Oguchi M, et al. $18 \mathrm{~F}-\mathrm{FDG}$ uptake as a biologic prognostic factor for recurrence in patients with surgically resected non-small cell lung cancer. $J$ Nucl Med. 2002;43:39-45.

30. Patz EF Jr, Connolly J, Herndon J. Prognostic value of thoracic FDG PET imaging after treatment for non-small cell lung cancer. AJR Am J Roentgenol. 2000;174:769-74.

\section{Discussion}

Dr Robert J. Cerfolio (Birmingham, Ala). This topic is both clinically important and relevant. I think that as general thoracic surgeons, we are going to see more and more patients who are undergoing neoadjuvant therapy, and we are going to be faced with the increasing challenge of choosing who is best brought to the surgical arena for attempted curative resection after therapy, and that decision is going to be based on what are relatively inaccurate restaging modalities. We do not believe the most recent article in The New England Journal of Medicine in January that showed that a very, very modest $5 \%$ increase in survival with adjuvant chemotherapy is going to swing the pendulum away from neoadjuvant therapy but rather toward more adjuvant therapy. Thus I think this article is important.
For time's sake, I am going to get right to my questions. I will give them to you one at a time. I have 3 sets of questions, but they are on just 3 basic topics. As you know, and you and I have talked about this, we have some data showing that the maximum SUV is an independent and better predictor of survival than the current TNM classification system for lung cancer. In addition, we have found that the percentage change in the maximum SUV on a repeat PET is a predictor of pathologic response, prognosis, and survival, with an $80 \%$ decrease in the maximum SUV after neoadjuvant therapy as a marker of complete pathologic response. In your article you examined the absolute value of the repeat PET with the SUV. Thirty-three of your 47 patients had an initial PET. Can you tell us whether you examined the percentage change in the maximum SUV, and did it correlate with the complete response rate, survival, or both?

Dr Graeter. Thank you for your kind remarks. We had a preinduction therapy PET in about $75 \%$ of the patients, and 25 of those 37 underwent operations. For those patients with nonviable tumors, the reduction was about $85 \%$. We then compared those with a reduction of more than $80 \%$ and those with a reduction of less than $80 \%$, but although the difference was about $40 \%$ in survival at 4 years, this did not reach a significant level.

Dr Cerfolio. Several reports have shown that a repeat PET scan can be difficult to interpret, especially if it is performed too soon after neoadjuvant radiotherapy, for example, within 2 or 3 weeks, and also that the higher the dose of the preoperative radiation, the more difficult it can be to interpret a repeat PET scan. Did you examine the accuracy of repeat PET scanning for different doses of radiotherapy, and can you tell us what you believe is the best time frame for performing a repeat PET scan after the completion of neoadjuvant therapy, especially radiotherapy?

Dr Graeter. All of these patients were acquired from ongoing induction therapy studies, and therefore they had a fairly uniform dose of between 50 and 55 Gy. Therefore the study was not really useful at differentiating whether 50 Gy is better than 55 Gy. But what we do know and what is published is that within the first 2 weeks, radiation leaves a lot of inflammatory changes, and that makes a PET scan very difficult to interpret. After 4 weeks, we hardly see any problems with that.

Dr Cerfolio. Do you recommend 4 weeks?

Dr Graeter. We recommend 4 weeks. Also, we had 2 groups, one with radiation and one with chemotherapy only, and radiation did not make any difference in the sensitivity parameters later on after 4 weeks.

Dr Cerfolio. My final question concerns the high number of patients with stage IIIB disease in this article, and I want to specifically focus on those who had N3 disease. It is a little different than most of our manuscripts would be. Many of us believe that recalcitrant $\mathrm{N} 2$ or N3 disease is a relative contraindication for thoracotomy and resection, as Dr Miller mentioned in the previous paper. Do you share this opinion, and if you do, how do you test for recalcitrant N3 disease? Do you do repeat mediastinoscopy, repeat endoscopic ultrasonography, or repeat videoassisted thoracoscopic surgery, or was the repeat PET scan and the change in the maximum SUV taken as the gospel truth? If so, then the contralateral lymph node really was never pathologically examined because you were in a different chest. 
Dr Graeter. This is a difficult question. Repeat mediastinoscopy is associated with a sensitivity of about $50 \%$ and an NPV that is equally poor, as published in the Annals about 2 years ago. We believe that in PET, if we have a low SUV in the contralateral lymph node and we do not have any sign of progressive disease, we will go ahead and operate.

Dr Mark J. Krasna (Baltimore, Md). Just to follow up on Dr Cerfolio's point, it would seem to me at this point premature to preclude somebody from undergoing surgical intervention if a PET scan was positive unless you proved it was positive pathologically. I would like to reiterate that I think it would also be premature to assume in somebody with an N3 tumor that it is okay to go ahead and do the resection because their PET results are now negative. As you showed, there are a significant number of false-negative and false-positive results. In those 2 scenarios I think you need to take that into consideration. Our policy has been to do contralateral video-assisted thoracoscopic surgery for those patients who have suspected N3 disease with an excellent yield. If you do not want to do a remediastinoscopy on those patients who have a persistent positive or suspicious spot on the PET scan, one should consider whether you want to actually restage that patient surgically before going ahead with a resection. Again, the pictures were beautiful, but if you have one single dot left out of those four, you need to make sure that that is the one you are going to sample. If you just sample the higher one or the lower one and that was the negative one, you might be missing the important finding.

My last comment goes to an interesting paper that came out last year from Europe on esophageal cancer. I wonder whether you have had any experience doing a repeat PET scan after the first cycle of chemotherapy in patients with neoadjuvant chemotherapy for esophageal cancer. This ended up being a predictor in that institution for outcome and complete pathologic response rate. Because not all of your patients had a PET pretreatment, did any of them have another PET scan before that final one, or was it just one at the very end?

Dr Graeter. For this analysis, we only used postinduction therapy PET scanning, but we did a multivariate analysis with TNM stage, type of tumor, radiation, yes, no, everything you can think of, and the only thing that really came out of it significantly was an SUV of greater than or less than 4 as a prognostic parameter for this group. In addition to your comment, if a PET scan is positive, we think that you always have to perform a biopsy before you take the next step. That is very important.

Dr Raphael Bueno (Boston, Mass). Our distribution of lung cancers in North America is a little different than in Europe in that you have more squamous carcinomas and we have more adenocarcinomas. Has the incorrect PET prediction that you have reported been mostly in any particular subtype of lung cancer, or were the patient numbers insufficient to determine that issue?

Dr Graeter. Actually, the only type we really have problems with is bronchoalveolar carcinoma, and that is well known for the primary staging, too.

Dr Nasser K. Altorki (New York, NY). My colleagues and I have just recently looked at the value of PET after induction chemotherapy and found that the NPV was very poor. The obvious concern here is that a negative PET scan after induction chemotherapy does not necessarily equal a complete pathologic response, yet that is precisely how it is generally perceived in the radiation and medical oncology worlds. I was wondering whether you found that the NPV differed whether your therapy was preoperative chemotherapy or preoperative chemoradiation. Did you look at that?

Dr Graeter. No, I did not. The values were almost the same. That is why I did not bring them up here. There was not much difference.

Dr David J. Sugarbaker (Boston, Mass). We have been interested at the Brigham in the ability to restage surgically patients treated with induction therapy, and there was a trial published some years ago from the CALGB, one of our clinical trials groups, that was a trial of patients with positive IIIA disease determined by means of mediastinoscopy, induction chemotherapy only, followed by surgical intervention, and when we analyzed the data, the patients who were living a long time were those who had been downstaged, whose mediastinal nodes once proved positive by means of biopsy had now become negative. We thought that was a marker of the biologic response of that tumor to the chemotherapy. That was about $30 \%$ of the patients who had been downstaged with this particular chemotherapy regimen, and therefore the majority had not been. I wonder whether you could comment on the role of radiotherapy as a potential confounder of the interpretation of mediastinal nodes after induction therapy in the use of PET in the way that you have described.

Dr Graeter. Radiation therapy did not have any influence on the outcome, irrespective of whether patients received radiation therapy. It also did not matter whether patients had any viable tumor left.

Dr Sugarbaker. I am not talking about the primary tumor. I am talking about the lymph nodes, the mediastinal nodes. Therefore, it did not matter in terms of prognosis whether there was residual tumor in the mediastinal nodes, is that what you said?

Dr Graeter. I was talking about the primary tumor right now.

Dr Sugarbaker. Okay.

Dr Graeter. The lymph nodes, it does matter, but again, it did not come out as a significant prognostic factor in this smaller group.

Dr Bueno. What was the long-term survival in the IIIB group?

Dr Graeter. It was about $65 \%$ if the SUV was less than 4.

Dr Bueno. But how long? What is the median survival?

Dr Graeter. For the 16 patients that had SUVs of less than 4, it was 57 months. For the IIIB group, it was about 55 .

Dr Cerfolio. I will just make a comment. If Dr Miller at the Southern accepts our abstract, we have some data that the amount of radiation does indeed make a difference, and we do not predict the mediastinal lymph nodes as well when we are at $60 \mathrm{~Gy}$ or greater, but at 45 Gy we do, and we do not predict them as well if we do a second PET scan within 3 weeks of the completion of radiation. Therefore we think the dose does make it more difficult, but again, that is a small series.

Dr Sugarbaker. Micrometastatic disease is what kills these patients, you know, distant disease. Therefore, if you sterilize the mediastinum, then the use of the mediastinal nodes reverting to negative as an indicator or sentinel of the effectiveness of chemotherapy on distant micrometastatic disease is gone. Then everybody looks like they are downstaged, but then you are still operating on the wrong patients because the majority of patients with IIIA and IIIB disease die of distant disease. That was the point. 